\title{
Update on Open Universe Inflationary Models
}

\author{
J.D. Cohn \\ Depts of Physics and Astronomy, University of Illinois at Urbana-Champaign, \\ Urbana, IL 61801
}

\begin{abstract}
An overview of some new results in open inflation over the past year, including the calculation of gravity wave contributions to the Cosmic Microwave Background.
\end{abstract}

In the past year, there has been significant progress in the understanding of the predictions and properties of open universe inflation. For backgrounf on earlier work and an introduction to these models see the original papers reviews 3 and references therein. Some key features of the earlier work are the following. Open universe inflationary models nucleate a bubble during inflation to generate curvature. The amount of inflation after nucleation of the bubble determines $\Omega$, usually put in by hand (but there are exception $\mathbf{3}^{3}$ ). Modifications of the original model include two field models 4 where one field generates inflation and one field nucleates the bubble. The Cosmic Microwave Background $(\mathrm{CMB})$ temperature fluctuations for scalars include both subcurvature and supercurvature scale perturbations, with the latter enhanced when the ratio of false to true vacuum energies is large. In the absence of gravity there was in addition a mode corresponding to fluctuations of the bubble wall itself (see the references in above reviews). Parameters such as properties of the false vacuum before tunneling and the wall profile have been varied in these models. These variations only influenced CMB temperature predictions on very large angular scale, changing only the overall normalization for open model large scale structure predictions

Recent developments include the calculation of gravity waves (tensor fluctuations of the background metric) for open universe models. Earlier unpublished work by Allen and Caldwell found an infinite CMB contribution from gravity waves originating in the natural de Sitter invariant vacuum. Defining

$$
\frac{\delta T(\hat{n})}{T} \frac{\delta T\left(\hat{n}^{\prime}\right)}{T}=\frac{1}{4 \pi} \sum_{\ell}(2 \ell+1) C_{\ell} P_{\ell}(\cos \theta)
$$

with $\hat{n} \cdot \hat{n}^{\prime}=\cos \theta$, they found $C_{\ell}=\infty$ for all $\ell$, all angular scales. For open universes created via bubble nucleation, the gravity waves originate in the de Sitter invariant (false) vacuum, but travel through the bubble wall to reach us. The energy difference agross the bubble wall regulates the divergence found by Allen and Caldwell 6 There have been efforts to regulate this divergence 
without introducing a bubble wall, unsuccessful so far 10 Thus, given our current understanding of the vacuum, the bubble is needed not only to provide the open universe but to make sure that the open universe has finite gravity waves, and any non-bubble open universe scenario needs some other mechanism to create finite gravity waves.

A second property of the gravity waves is that the lowest momentum gravity wave contribution is degenerate with the 'would-be' wall mode, the bubble wall fluctuations in the absence of gravity. This combined mode seems non-normalizable. Although not completely conclusive, a rigorous and detailed quantization of the coupled scalar field and gravity system supports the absence of these modes as well 11 In the coupled gravity wave and bubble wall system the bubble wall oscillates as the gravity waves go across it 12 A relation between gravity waves and would-be wall fluctuations is also useful for a heuristic description of the CMB gravity wave spectrum (for chaotic inflation models inside the bubble), where the tensor contribution can be written as a "residual" plus a "wall" contribution 13

$$
C_{\ell}^{T}=\frac{1}{H_{T}^{2}} \tilde{C}_{\ell}^{T, \min }(\Omega)+\frac{A(\text { model })}{H_{T}^{2}} \tilde{C}_{\ell}^{\text {wall }}(\Omega), H_{T}=\text { Hubble constant } .
$$

There is a minimal gravity wave contribution 14 with the gravity waves suppressed by a factor of $V^{\prime} / V$ relative to the sfalars. The tensor to scalar ratio has dependence on $\Omega$ and model parameters 15 unlike flat models. As the gravity wave spectrum can be steep at low $\ell$, and large, requiring agreement with observations constrains models. Parameter ranges for theories satisfying the gravity wave constraints were given for chaotic inflation 13 and fon recently proposed models of tilted open hybrid inflation 16 and dilaton/scala 17 inflation. Open model scalar and tensor contributions can now be calculated with the publicly available Boltzmann code CMBFAST18 19 (which includes the four year COBE normalization 20). The CMB polarization gives additional information and has been calculated for some open models 19

Another area of study was the properties of the open universe backgrounds. In the simplest two field models 1 inhomogeneities can arise from the evolution of the second field while the first field is driving inflation21 In addition 22 the supercurvature modes in several two field models destroy the background homogeneity, restricting the second stage of inflation to finite regions inside the bubble. Calculating the observationally viable range of parameters for these quasi-open models is in progress 22 Remaining questions for these models include the likelihood of motivating the appropriate field theory potentials from a high energy physics theory such as string theory.

I thank A. Liddle and M. White for conversations and the organizers for 
a stimulating and exciting conference. This work was supported by an ONR grant and nsf-phy9722787.

1. In particular, M. Bucher, A.S. Goldhaber, N. Turok, Phys. Rev. D 52, 3314 (1995), hep-ph/9411206; see also K. Yamamoto, M. Sasaki, T. Tanaka, ApJ 455, 412 (1995);

2. J. Garcia-Bellido, Contribution to TAUP '95 Conference Proceedings, Toldeo, Sept. 1995, astro-ph/9511078; J. D. Cohn, in Microwave Background Anisotropies, Eds. Bouchet et al, Editions Frontieres, 1997, France, astro-ph/9606052.

3. A. Vilenkin, S.Winitzki, Phys. Rev. D 55, 548 (1997), astro-ph/9605191

4. A. D. Linde, Phys. Lett. B 351, 99 (1995), hep-th/9503097; A. D. Linde, A. Mezhlumian, Phys. Rev. D 55, 609 (1997), astro-ph/9506017

5. M. Sasaki, T. Tanaka, K. Yamamoto, Phys. Rev. D 51, 2979 (1995), gr-qc/9412025

6. M. Sasaki, T. Tanaka, Phys. Rev. D 54, 4705 (1996), astro-ph/9605104

7. A. Liddle, P. Viana, MNRAS 281, 323 (1996), astro-ph/9511007; M. White, J. Silk, Phys. Rev. Lett. 77, 4704 (1996); Erratum Phys. Rev. Lett. 78, 3799 (1997),astro-ph/9608177; K. Gorski et al, ApJS 114, 1 (1998), astro-ph/9608054

8. T. Tanaka, M. Sasaki, Prog. Theor. Phys. 97, 243 (1997), astroph/9701053;

9. M. Bucher, J. D. Cohn, Phys. Rev. D 55, 7461 (1997), astro-ph/9701117

10. R. Caldwell, private communication.

11. J. Garriga, X. Montes, M. Sasaki, T. Tanaka, "Canonical Quantization of Cosmological Perturbations in the One Bubble Open Universe," astro$\mathrm{ph} / 9706229$

12. A. Ishibashi and H. Ishihara,Phys. Rev. D 56, 3446 (1997); A. Ishibashi, H. Ishihara, "On the perturbation of domain wall coupled to gravitational waves," gr-qc/9710077

13. M. Sasaki, T. Tanaka, Y. Yakushige, Phys. Rev. D 56, 1997 (616),astro$\mathrm{ph} / 9702174$

14. W. Hu, M. White, ApJ 486, L1 (1997), astro-ph/9701210

15. J. Garcia-Bellido, Phys. Rev. D 56, 3225 (1997), astro-ph/9702211

16. J. Garcia-Bellido, A. Linde, Phys. Rev. D 55, 7480 (1997), astro$\mathrm{ph} / 9701173$

17. A. Green, A. Liddle, Phys. Rev. D 55, 609 (1997), astro-ph/9607166; J. Garcia Bellido, A. Liddle, Phys. Rev. D 55, 4603 (1997), astro$\mathrm{ph} / 9610183$

18. U. Seljak, M. Zaldariagga, ApJ 469, 437 (1996), astro-ph/9603033; M. Zaldariagga, U. Seljak, E. Bertschinger, "Integral Solution for 
the Microwave Background Anisotropies in Non-flat Universes," astro$\mathrm{ph} / 9704265$

19. W. Hu, U. Seljak, M. White, M. Zaldariagga, "A Complete Treatment of CMB Anisotropies in a FRW Universe," astro-ph/9709066

20. E. Bunn, M. White, ApJ 480, 6 (1997), astro-ph/9607060

21. J. Garriga, V. Mukhanov, Phys. Rev. D 56, 2439 (1997), astro$\mathrm{ph} / 9702201$

22. J. Garcia-Bellido, J. Garriga, X. Montes, "Quasi-open inflation", hep$\mathrm{ph} / 9711214$. 